\title{
Nanostructure thermal conductivity prediction by Monte Carlo sampling of phonon free paths
}

\author{
Alan J. H. McGaughey a) and Ankit Jain \\ Department of Mechanical Engineering, Carnegie Mellon University, Pittsburgh, Pennsylvania 15213, USA
}

(Received 9 December 2011; accepted 21 January 2012; published online 9 February 2012)

\begin{abstract}
We propose a method by which the thermal conductivity of a nanostructure with arbitrary geometry can be predicted through Monte Carlo sampling of the free paths associated with phonon-phonon and phonon-boundary scattering. The required inputs are the nanostructure geometry and the bulk phonon frequencies, group velocities, and mean free paths. The method is applied to a thin film in the in-plane and cross-plane directions and to a polycrystalline bulk material. For the film, a faster approach to the bulk thermal conductivity is found compared to predictions made using the Matthiessen rule with the bulk mean free path and an average phonon-boundary scattering length. (C) 2012 American Institute of Physics. [doi:10.1063/1.3683539]
\end{abstract}

As the dimensions of electronic, optoelectronic, and energy conversion devices are reduced, the thermal conductivities of the device components (e.g., thin films and nanowires) are also reduced. ${ }^{1-11}$ The large electrical power densities in such devices cause Joule heating and the reduced thermal conductivities can lead to high operating temperatures, sub-optimal performance, and poor reliability. Predicting the thermal conductivity reduction in nanostructures is thus a critical part of developing next-generation thermal management strategies.

We focus here on semiconducting and insulating nanostructures, where phonons (quantized lattice vibrations) dominate thermal transport. ${ }^{12}$ As a nanostructure gets smaller, its thermal conductivity is reduced due to more frequent scattering between phonons and the system boundaries. ${ }^{1-11}$ For very small systems (e.g., silicon films thinner than $20 \mathrm{~nm}$ ), changes in the phonon density of states also affect thermal transport. ${ }^{2,6,8}$ Our interest here is nanostructures large enough that the phonon density of states is bulk-like.

The thermal conductivity, $k$, of a nanostructure in the $n$ direction can be predicted from, ${ }^{12}$

$$
k_{n}=\sum_{i} c_{p h, i} v_{g, n, i}^{2} \frac{\overline{\Lambda_{i}}}{\left|\mathbf{v}_{g, i}\right|},
$$

an expression obtained by solving the Boltzmann transport equation under the relaxation time approximation and using the Fourier law. The summation is over all phonon modes in the first Brillouin zone, $c_{p h, i}$ is the mode volumetric specific heat, $v_{g, n, i}$ is the $n$ component of the group velocity vector, $\mathbf{v}_{g i}$, and $\overline{\Lambda_{i}}$ is the mean free path. The mean free path is the average distance a phonon travels before scattering and combines the effects of all scattering mechanisms (e.g., with other phonons, boundaries, defects, electrons, etc.).

Phonon scattering in a semiconductor or insulating nanostructure at room temperature and above is dominated by phonon-phonon and phonon-boundary interactions. The standard approach for combining these two effects is to use the Matthiessen rule, whereby the scattering mechanisms are

${ }^{a)}$ Electronic mail: mcgaughey@cmu.edu. assumed to be independent, such that the mean free path is given by, ${ }^{12}$

$$
\frac{1}{\overline{\Lambda_{i}}}=\frac{1}{\overline{\Lambda_{p p, i}}}+\left(\frac{1-p_{i}}{1+p_{i}}\right) \frac{1}{\bar{l}_{i}} .
$$

Here, $\overline{\Lambda_{p p, i}}$ is the bulk phonon-phonon mean free path and $\bar{l}_{i}$ is the average distance a phonon travels ballistically before hitting a system boundary with mode-dependent specularity parameter $p_{i}$. Phonon-boundary scattering at room temperature and above is expected to be predominantly diffuse. ${ }^{13}$ While we take $p_{i}=0$ in the analysis that follows, the proposed technique can be used for any value of $p_{i}$.

The two terms on the right-hand side of the Matthiessen rule, Eq. (2), are averages. The mean free path associated with phonon-phonon scattering is an average over many collisions, each with its own free path, $\Lambda_{p p i}$. The mean free path associated with phonon-boundary scattering is based on a single length for each mode (e.g., $\frac{L}{2}\left|\frac{\mathbf{v}_{g, i}}{v_{g, z, i}}\right|$ for a film of thickness $L$ that is normal to the $z$ direction ${ }^{11}$ ). The phonon can in fact start at any position in the nanostructure. The goal of this study is to determine if the thermal conductivity predicted using Eq. (2) is the same thermal conductivity that results from considering the natural distributions of phononphonon and phonon-boundary free paths.

We now propose a Monte Carlo method for including free path distributions in nanostructure thermal conductivity prediction based on knowledge of the bulk phonon properties and the system geometry. Bulk phonon-phonon mean free paths can be predicted for a fine resolution of the first Brillouin zone using molecular dynamics simulations ${ }^{14-16}$ and/or lattice dynamics calculations, ${ }^{14,16-19}$ with input from empirical potentials ${ }^{15,16,20}$ or first-principles density functional theory calculations. ${ }^{17,19,21}$ The geometry of a nanostructure can be resolved to subnanometer scales using electron microscopy techniques, allowing for accurate inclusion in calculations.

As a test system, we choose a silicon thin film at a temperature, $T$, of $300 \mathrm{~K}$ with bulk phonon properties calculated using the Stillinger-Weber potential ${ }^{22}$ (see Fig. 1). Both the in-plane and cross-plane thermal conductivities are predicted. The bulk phonon frequencies, group velocities, and 


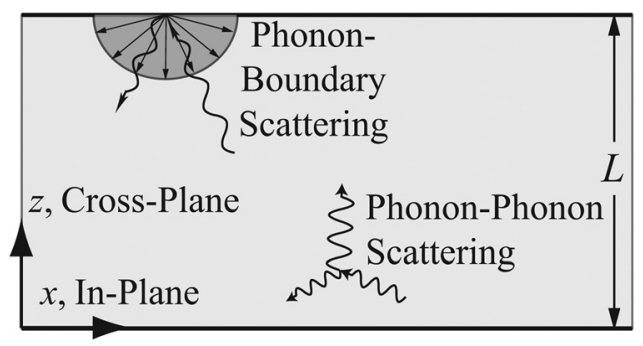

FIG. 1. Film model. The film is infinite in the in-plane directions.

mean free paths are calculated using harmonic and anharmonic lattice dynamics calculations with Bose-Einstein (i.e., quantum) statistics. We previously reported on predictions of Stillinger-Weber silicon bulk and thin film thermal conductivities [with Eq. (2) used to include the effects of boundary scattering]. ${ }^{6,7}$ A first Brillouin zone with 55572 phonon modes (9262 evenly spaced wave vectors with six phonon modes/wave vector) was found to be sufficient to predict a size-independent bulk thermal conductivity and is used here.

We first loop over the 55572 phonon modes in the first Brillouin zone. For each mode, the following procedure is repeated 1000 times:

1. A phonon-phonon free path is randomly drawn from a Poisson distribution, $P\left(\Lambda_{p p, i}\right)$, given by,

$$
P\left(\Lambda_{p p, i}\right)=\frac{1}{\overline{\Lambda_{p p, i}}} \exp \left(-\Lambda_{p p, i} / \overline{\Lambda_{p p, i}}\right) .
$$

The Poisson distribution is chosen as it describes the free path distribution of the particles in an ideal gas. ${ }^{23}$ As we are modeling the phonons as particles (i.e., a phonon gas), this choice seems reasonable.

2. A point is randomly chosen in the nanostructure. For the film, this choice amounts to choosing a value of $z$ between 0 and $L$. Using the phonon mode's group velocity vector, which gives the direction of energy propagation, the distance the phonon will travel ballistically before hitting a boundary is calculated. For diffuse boundary scattering, this distance is the phononboundary free path, $l$. If the boundary scattering is partially specular (i.e., $p_{i} \neq 0$ ), $l$ can be calculated by considering a series of elastic reflections and ballistic paths through the nanostructure [see second term on the right-hand side of Eq. (2)].

3. The minimum of the two free paths, $\Lambda_{p p}$ or $l$, is taken as the nanostructure free path. This choice removes the assumption in the Matthiessen rule that the scattering mechanisms are independent.

The 1000 free paths are averaged to give the mean free path for that mode. Once this procedure has been performed for all modes, Eq. (1) is applied to predict thermal conductivity. We find that 1000 free paths/mode is sufficient to predict a thermal conductivity within $1 \%$ of the value obtained using 10000 free paths/mode and that this error decreases with increasing film thickness. Running ten separate calculations with 1000 free paths/mode gives a standard deviation of less than $0.5 \%$ of the mean thermal conductivity.

The in-plane and cross-plane thermal conductivity predictions for film thicknesses between $20 \mathrm{~nm}$ and $10 \mu \mathrm{m}$ are plotted in Fig. 2. Also plotted are the thermal conductivities predicted by a direct use of the Matthissen rule [Eq. (2)] in Eq. (1) (i.e., no random sampling). All the film thermal conductivities are normalized by the bulk value, $k_{\infty}$, and approach it as the film thickness increases.

Calculating the mean free path with random sampling of the phonon-phonon and phonon-boundary free paths leads to a faster approach to the bulk thermal conductivity than using average values of these two quantities and the Matthiessen rule. The maximum difference is $6 \%$ for the in-plane direction and $10 \%$ for the cross-plane direction for film thicknesses around $500 \mathrm{~nm}$. This thickness corresponds to about twice the average specific heat- and group velocity-weighted mean free path in SW silicon at a temperature of $300 \mathrm{~K}$ $(243 \mathrm{~nm})$ (Ref. 6) and is thus where phonon-phonon and phonon-boundary scattering effects are comparable.

For a film with a temperature gradient in the in-plane direction, Turney et al. solved the Boltzmann transport equation without invoking the Matthiessen rule and found that the mean free path is given by, ${ }^{6}$

$$
\overline{\Lambda_{i}}=\left[1-\frac{1-p_{i}}{\delta_{i}} \frac{1-\exp \left(-\delta_{i}\right)}{1-p_{i} \exp \left(-\delta_{i}\right)}\right] \overline{\Lambda_{p p, i}},
$$

where $\delta_{i}=\frac{L}{\Lambda_{p p, i}}\left|\frac{\mathbf{v}_{g, i}}{v_{g, z i}}\right|$. When we use Eq. (4) with $p_{i}=0$ in Eq. (1) to predict thermal conductivity, we get a value within $1 \%$ of that predicted using the random sampling technique with no systematic error. This finding indicates that these two approaches are capturing the same physics and highlights the limitations of the Matthiessen rule for combining phonon-phonon and phonon-boundary scattering.

For more complicated geometries (e.g., porous silicon meshes ${ }^{9}$ ), it is not obvious what single length scale could be used for the phonon-boundary scattering mean free path in the Matthiessen rule. Furthermore, an exact solution of the Boltzmann transport equation, providing an expression like Eq. (4), may not be possible. Our free path Monte Carlo sampling technique removes these complications.

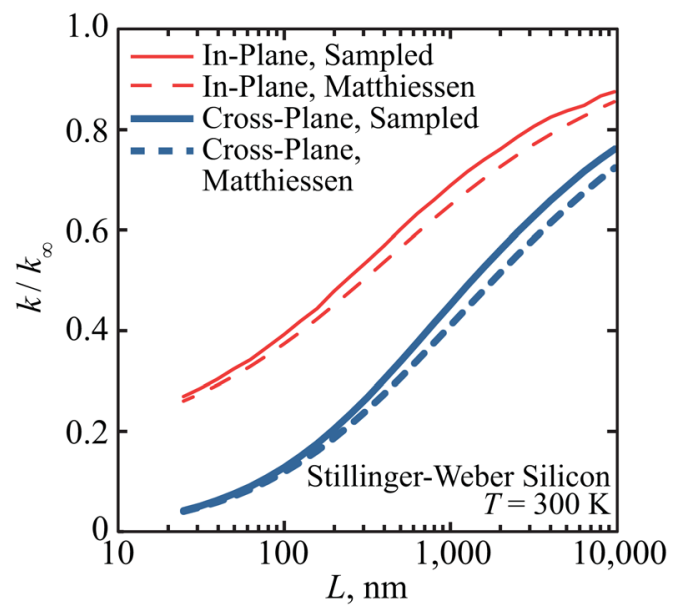

FIG. 2. (Color online) Thin film thermal conductivity predictions for inplane and cross-plane directions by Matthiessen rule and sampled phonon mean free paths. The curves are generated by considering ten film thickness per decade. By properly sampling the phonon-phonon and phonon-boundary free paths, a larger thermal conductivity and faster approach to the bulk thermal conductivity are predicted compared to the average values used in the Matthiessen rule. 


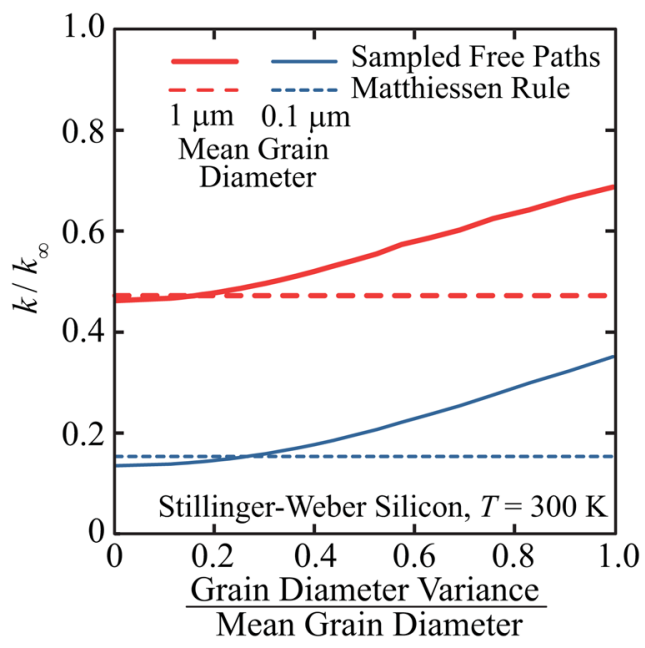

FIG. 3. (Color online) Thermal conductivity of polycrystalline StillingerWeber silicon as a function of the mean grain diameter and the grain diameter variance. For the solid lines, the grain diameter, phonon-phonon free path, and phonon starting point are all randomly sampled. The dashed lines correspond to predictions made using the Matthiessen rule with the bulk mean free paths, one geometric scattering length, and the mean grain diameter.

To demonstrate the usefulness of our technique, we now predict the thermal conductivity of polycrystalline bulk Stillinger-Weber silicon at a temperature of $300 \mathrm{~K}$. Typically, the average grain size is used in the Matthiessen rule to include the effect of phonon-grain boundary scattering. ${ }^{24,25}$ We use spherical grains for computational efficiency, but the grain shape can be arbitrary. To calculate the mean free path of each phonon mode, we randomly sample three quantities. First, a grain diameter is selected from a log-normal distribution. ${ }^{26}$ Second, a point is randomly chosen in the grain, allowing for calculation of the phonon-boundary free path (we again assume diffuse boundary scattering). Third, a phonon-phonon free path is selected from a Poisson distribution based on the bulk mean free path. The nanostructure free path is taken as the smaller of the phonon-boundary and phonon-phonon free paths. The mean free path for that mode is calculated by 1000 such samplings of grain diameter, initial position, and phonon-phonon free path, and weighting the contributions to thermal conductivity by the grain volume. The resulting thermal conductivities for average grain diameters of $100 \mathrm{~nm}$ and $1 \mu \mathrm{m}$ are plotted as a function of the grain diameter variance divided by the mean grain diameter in Fig. 3. Also plotted is the thermal conductivity predicted using the Matthiessen rule (i.e., using the bulk phonon mean free paths, one geometric scattering length, and the mean grain diameter). As the variance in the grain diameter increases, assuming a single grain diameter can lead to a significant underprediction of thermal conductivity. We note that our predictions should be interpreted qualitatively, as the thermal conductivity of a polycrystalline material will depend on the grain shapes and relative orientations.

The proposed calculation technique runs quickly (given a set a phonon properties, on the order of seconds for each thermal conductivity prediction described here), can access much larger length scales than atomistic calculations, and better captures the phonon physics compared to using the Matthiessen rule. As such, we recommend that it be used for predicting the thermal conductivity of other nanostructures. The major assumptions in our implementation of the free path Monte Carlo sampling technique are that (i) the bulk phonon-phonon free paths follow a Poisson distribution and that this distribution will hold in a nanostructure, (ii) a phonon is equally likely to originate at any point in the nanostructure, and (iii) the bulk phonon properties (frequency, group velocity, and mean free path) are valid everywhere in the nanostructure. Detailed theory or experimental results do not exist to test these assumptions. While we believe that our assumptions are reasonable, their study is an intriguing direction for future efforts.

This work was supported by AFOSR award FA95501010098. We thank Daniel P. Sellan (University of Toronto) for supplying the bulk phonon properties and for helpful discussions.

${ }^{1}$ M. Asheghi, Y. K. Leung, S. S. Wong, and K. E. Goodson, Appl. Phys. Lett. 71, 1798 (1997).

${ }^{2}$ A. Balandin and K. L. Wang, Phys. Rev. B 58, 1544 (1998).

${ }^{3}$ D. Li, Y. Wu, P. Kim, L. Shi, P. Yang, and A. Majumdar, Appl. Phys. Lett. 83, 2934 (2003).

${ }^{4}$ A. I. Hochbaum, R. Chen, R. D. Delgado, W. Liang, E. C. Garnett, M. Najarian, A. Majumdar, and P. Yang, Nature 451, 163 (2008).

${ }^{5}$ P. Martin, Z. Aksamija, E. Pop, and U. Ravaioli, Phys. Rev. Lett. 102, 125503 (2009).

${ }^{6}$ J. E. Turney, A. J. H. McGaughey, and C. H. Amon, J. Appl. Phys. 107, 024317 (2010).

${ }^{7}$ D. P. Sellan, J. E. Turney, A. J. H. McGaughey, and C. H. Amon, J. Appl. Phys. 108, 113524 (2010).

${ }^{8}$ J.-K. Yu, S. Mitrovic, D. Tham, J. Varghese, and J. R. Heath, Nature Nanotechnol. 5, 718 (2010).

${ }^{9}$ P. E. Hopkins, C. M. Reinke, M. F. Su, R. H. Olsson, E. A. Shaner, Z. C. Leseman, J. R. Serrano, L. M. Phinney, and I. El-Kady, Nano Lett. 11, 107 (2011).

${ }^{10}$ Z. Tian, K. Esfarjani, J. Shiomi, A. S. Henry, and G. Chen, Appl. Phys. Lett. 99, 053122 (2011).

${ }^{11}$ A. J. H. McGaughey, E. S. Landry, D. P. Sellan, and C. H. Amon, Appl. Phys. Lett. 99, 131904 (2011).

${ }^{12} \mathrm{~J}$. M. Ziman, Electrons and Phonons (Oxford, New York, 2001).

${ }^{13}$ J. C. Duda, T. E. Beechem, J. L. Smoyer, P. M. Norris, and P. E. Hopkins, J. Appl. Phys. 108, 073515 (2010).

${ }^{14}$ A. J. C. Ladd, B. Moran, and W. G. Hoover, Phys. Rev. B 34, 5058 (1986).

${ }^{15}$ A. J. H. McGaughey and M. Kaviany, Phys. Rev. B 69, 094303 (2004).

${ }^{16}$ J. E. Turney, E. S. Landry, A. J. H. McGaughey, and C. H. Amon, Phys. Rev. B 79, 064301 (2009).

${ }^{17}$ D. A. Broido, M. Maloney, G. Birner, N. Mingo, and D. Stewart, Appl. Phys. Lett. 91, 231922 (2007).

${ }^{18}$ A. Chernatynskiy and S. R. Phillpot, Phys. Rev. B 82, 134301 (2010).

${ }^{19}$ K. Esfarjani, G. Chen, and H. T. Stokes, Phys. Rev. B 84, 085204 (2011).

${ }^{20}$ A. S. Henry and G. Chen, J. Comput. Theor. Nanosci. 5, 1 (2008).

${ }^{21}$ J. Garg, N. Bonini, B. Kozinsky, and N. Marzari, Phys. Rev. Lett. 106, 045901 (2011).

${ }^{22}$ F. H. Stillinger and T. A. Weber, Phys. Rev. B 31, 5262 (1985).

${ }^{23}$ S. J. Jeans, An Introduction to the Kinetic Theory of Gases (Cambridge University Press, Cambridge, 1940).

${ }^{24}$ S. Uma, A. D. McConnell, M. Asheghi K. Kurabayashi, and K. E. Goodson, Int. J. Thermophys. 22, 605 (2001).

${ }^{25}$ Z. Wang, J. E. Alaniz, W. Jang, J. E. Garay, and C. Dames, Nano Lett. 11, 2206 (2011).

${ }^{26}$ R. B. Bergmann, F. G. Shi, H. J. Queisser, and J. Krinke, Appl. Surf. Sci. 123/124, 376 (1998). 